\title{
Localisation and disorder in the design of 2D photonic crystal devices
}

\author{
T. N. Langtry L. C. Botten A. A. Asatryan \\ M. A. Byrne* A. Bourgeois ${ }^{\dagger}$
}

(Received 8 August 2003, revised 16 February 2004)

\begin{abstract}
Photonic crystals are meta-materials that can inhibit the propagation of light in all directions for specific wavelength ranges. Material or structural defects can be introduced into the crystal to cause localised modes, providing the ability to mould the flow of light on the wavelength scale and allowing the development of miniaturised, integrated photonic devices. For this reason, photonic crystals will likely be key building blocks for future micro-optical and communication technology. In this paper, we examine the Bloch mode modelling of $2 \mathrm{D}$ photonic crystal structures with application to the analysis of photonic crystal waveguides and their susceptibility to disorder, which provides a framework for studying fabrication tolerances in realistic devices.
\end{abstract}

${ }^{*}$ CUDOS \& Dept. Math. Sciences, University of Technology, Sydney, Australia. mailto:Tim. Langtry@uts . edu . au

†Université de Paris-Sud, Bâtiment 470, 91405 Orsay Cedex, France.

See http://anziamj.austms.org.au/V45/CTAC2003/Lang/home.html for this article, (C) Austral. Mathematical Soc. 2004. Published July 30, 2004. ISSN 1446-8735 


\section{Contents}

1 Introduction

C745

2 Theoretical Formulation

2.1 Bloch mode modelling of extended PC devices . . . . . . C748

2.2 Photon Conductance . . . . . . . . . . . . . . .

3 Computational Results

C754

References

C757

\section{Introduction}

Photonic crystals (PCs) [5] are a novel type of complex material in which the refractive index varies periodically with position. Through interference action known as Bragg reflection, PCs can inhibit the propagation of electromagnetic waves for various ranges of frequencies, giving rise to "band gaps" in their spectrum. It is these that make PCs the optical analogues of semiconductors. Localised disruption of the periodicity of the index by the introduction of structured defects into the material allows controlled propagation of light at frequencies within a band gap. This capacity to tailor the propagation of light on the scale of optical wavelengths with minimal loss was a prime motivation for the advent of PCs $[6,11]$. It is the reason that PCs are likely to be a key building block for future micro-optical and communication technology, providing optical functionality analogous to that available in electronic chips and so facilitating signal processing at unprecedented speed.

Of current interest is the design of PC-based devices that will allow the miniaturization of photonic components, and the use of PCs to modify the radiation dynamics of sources embedded within them. Various devices and interconnections such as bends, junctions and filters have been modelled and 
fabricated [9]. The exploitation of the technological potential of PCs however requires a thorough understanding of the coupling and guiding mechanisms. Coupling problems, which are both challenging and general, involve interfacing various PC media and waveguide devices to one another or to some external medium. While most investigations to date have been largely computational, we have recently developed a new approach [2], based on Bloch modes and elaborated in Section 2.1. The method exploits the underlying physics and provides substantial analytic and computational advantages. It is based on earlier work [1,3] in which a method based on multipole expansions was developed for the modelling of both regular (periodic) and irregular or finite clusters. This method yields high computational speed and accuracy, and considerable analytic insight, albeit with some restrictions on the range of geometries that can be considered. As an example, we note the strong similarities that exist between photonic phenomena and their electronic counterparts and, in Section 2.2, transfer the concept of electron conductance, one of the main properties of nano-scale wires, to photonics.

The examples considered in this paper are based on a PC consisting of infinitely long cylinders arranged on a square lattice with lattice constant $d$. Each cylinder, in the regular case, has radius $0.3 d$ and refractive index 3.0. The background index is 1.0. This material has a bandgap at wavelengths in the range $3.0 d \leq \lambda \leq 3.7 d$. We also consider irregular cases in which some of the cylinder parameters are subject to random perturbations. In Section 3 we present results illustrating the effect of disorder in the refractive index of the cylinders on the conductance of photons in PC waveguides. We also observe that these results are consistent with the effect of similar disorder on electric field intensity in such guides. 


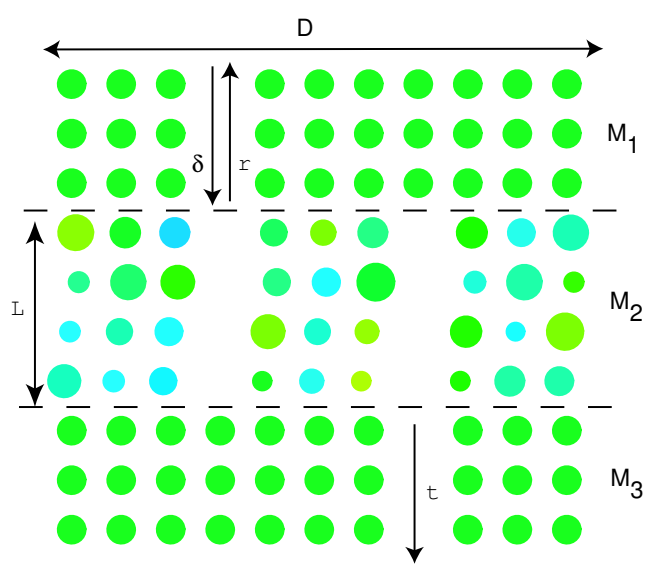

(a)

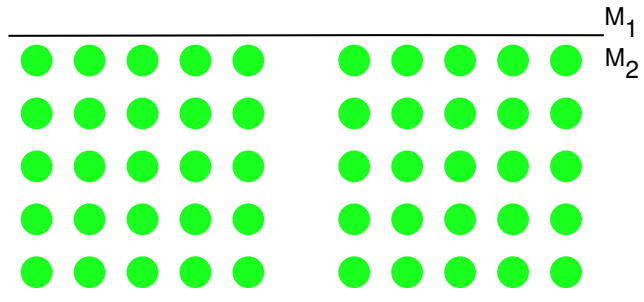

(b)

Figure 1: Device designs which may be seen as either finite clusters of scatterers or extended devices (when one or more regions is assumed to be semi-infinite in extent). (a) A directional coupler exhibiting disorder in the coupling region $\left(M_{2}\right)$. (b) Waveguide coupling to free space.

\section{Theoretical Formulation}

In this section, we outline a theoretical model for the diffraction of light by extended PC devices such as those shown in Figure 1.

When considered as a finite cluster of cylinders, the structure may be modelled using a multipole expansion method [1]. However, for an extended device, as in Figure 1, in which the front and rear channels (or waveguides) are arbitrarily long, a more elaborate analysis involving the basis of natural (Bloch) modes of the various structural elements is required. Section 2.1 treats a broad class of structures exemplified by waveguides and by more elaborate devices such as the folded directional coupler [12] of Figure 1(a), and in Section 2.2 we outline the theory for the quantification of photon conductance. 


\subsection{Bloch mode modelling of extended PC devices}

To date, much of the modelling of PC devices has been undertaken using exclusively computational tools such as finite difference time domain (FDTD) methods. While these produce reliable and useful results, they provide little theoretical and analytic insight into the underlying physics, and invariably, for devices of realistic size, require vast computational resources. For these reasons, we have sought to develop a suite of semi-analytic tools than can handle the wide range of coupling and guiding problems that arise in the design of PC devices in a highly efficient manner. While multipole methods are effective in modelling finite, compact structures, they are not a practical approach to dealing with extended structures such as that of Figure 1(a). Instead, we exploit plane wave methods as the underlying toolkit for coupling together the various elements and layers of PC devices, building on these to develop analytic and computational tools in the natural basis of Bloch modes of the structural elements. Specifically, we have developed a theory which efficiently separates the propagation in linear sections (for example, waveguides) from the scattering and diffraction of modes at interfaces between device segments. The latter is characterized by matrix generalisations of the Fresnel reflection and transmission coefficients that are used widely in thin film optics.

As an example, consider the propagation problem of Figure 1(a) in which three distinct media are jointed together. Each medium may be homogeneous (for example, free space or dielectric) or a periodically modulated structure such as a photonic crystal, possibly penetrated by waveguides as shown. Each medium is characterized by its Bloch modes, generated via a transfer matrix technique [4] based on supercell methods that exploits diffraction grating theories. Initially, we consider the region $M_{2}$ to comprise $L$ identical layers.

The Bloch modes which characterise propagation in a region $M_{s}$ are obtained by solving an eigenvalue problem derived by considering an infinite region comprising only layers of $M_{s}$. This infinite structure is unaltered by 
a translation of a single layer, and hence the electromagnetic fields one layer apart must be related by a simple multiplication factor $\mu=\exp \left(i k_{0 y} d\right)$ where $d$ is the interlayer spacing and $k_{0 y}$ is the relevant component of the Bloch vector $\mathbf{k}_{0}$. That is, $V(x, y+d)=\mu V(x, y)$. At the boundary of each layer $j$ (that is, midway between the line of centres of the cylinders), we express the fields in expansions of upward and downward propagating plane waves

$$
V(x, y)=\sum_{p=-\infty}^{\infty}\left[f_{p}^{(j)-} e^{-i \chi_{p}\left(y-y_{j}\right)}+f_{p}^{(j)+} e^{+i \chi_{p}\left(y-y_{j}\right)}\right] e^{i \alpha_{p} x}
$$

We use a supercell method with lateral period $D$ (Figure 1(a)) chosen to be sufficiently large so that there is negligible cross-talk between channels when the PC structure is operated in a band gap. In this case, the direction sines and cosines of the plane wave terms in (1) are given by $\alpha_{p}=k_{0 x}+2 \pi p / D$ and $\chi_{p}=\sqrt{k^{2}-\alpha_{p}^{2}}$, where $\mathbf{k}=\left(k_{0 x}, k_{0 y}\right)^{T}$ and $k=\|\mathbf{k}\|=2 \pi / \lambda$ is the wavenumber of the light of wavelength $\lambda$ in free space. Defining $\mathbf{f}_{j}^{\mp}=\left[f_{p}^{(j) \mp}\right]$ (vectors of plane wave coefficients), the scattering action of a single layer is

$$
\mathbf{f}_{2}^{-}=\mathbf{T f}_{1}^{-}+\mathbf{R f}_{2}^{+}, \quad \mathbf{f}_{1}^{+}=\mathbf{R f}_{1}^{-}+\mathbf{T f}_{2}^{+},
$$

expressing the outgoing fields as reflections and transmissions of the incoming fields. In (2), $\mathbf{R}$ and $\mathbf{T}$ denote reflection and transmission scattering matrices that characterise the diffraction properties of the cylinder grating layer. These may be computed using a variety of approaches derived from diffraction grating theory. In our case, we exploit multipole methods [3], tailored to periodic structures, to generate $\mathbf{R}$ and $\mathbf{T}$.

Solving (2) for $\mathbf{f}_{2}^{-}$and $\mathbf{f}_{2}^{+}$, we obtain an interlayer translation operator $\mathcal{T}$ from which follows the eigenvalue equation determining the Bloch modes.:

$$
\left[\begin{array}{c}
\mathbf{f}_{2}^{-} \\
\mathbf{f}_{2}^{+}
\end{array}\right]=\mathcal{T}\left[\begin{array}{l}
\mathbf{f}_{1}^{-} \\
\mathbf{f}_{1}^{+}
\end{array}\right]=\mu\left[\begin{array}{l}
\mathbf{f}_{1}^{-} \\
\mathbf{f}_{1}^{+}
\end{array}\right]
$$

where

$$
\mathcal{T}=\left[\begin{array}{cc}
\mathbf{T}_{s}-\mathbf{R}_{s} \mathbf{T}_{s}{ }^{-1} \mathbf{R}_{s} & \mathbf{R}_{s} \mathbf{T}_{s}^{-1} \\
-\mathbf{T}_{s}{ }^{-1} \mathbf{R}_{s} & \mathbf{T}_{s}{ }^{-1}
\end{array}\right]
$$


While (3) formally defines the modes, the truncated numerical problem involving $\mathcal{T}$ is not well conditioned, particularly for matrices of large dimension, and thus an alternative method [10] is needed to compute the modes.

Bloch modes occur in pairs, corresponding to forward and backward propagation, and may be classified either according to the direction of their energy flux in the case of the finite number of propagating modes $(|\mu|=1)$, or whether $|\mu| \lessgtr 1$ for the unbounded set of evanescent modes which carry no energy. In this way we diagonalise the operator $\mathcal{T}$, which for vertically symmetric structures (such as square or rectangular lattices) yields:

$$
\mathcal{T}=\left[\begin{array}{ll}
\mathbf{F}^{-} & \mathbf{F}^{+} \\
\mathbf{F}^{+} & \mathbf{F}^{-}
\end{array}\right]\left[\begin{array}{cc}
\boldsymbol{\Lambda} & \mathbf{0} \\
\mathbf{0} & \mathbf{\Lambda}^{-1}
\end{array}\right]\left[\begin{array}{cc}
\mathbf{F}^{-} & \mathbf{F}^{+} \\
\mathbf{F}^{+} & \mathbf{F}^{-}
\end{array}\right]^{-1}
$$

where the matrices $\mathbf{F}^{\mp}$ comprise columns of eigenvector components $\mathbf{f}_{j}^{\mp}$ associated with forward propagating modes with eigenvalues $\boldsymbol{\Lambda}=\operatorname{diag}\left\{\mu_{j}\right\}$. In (5), the left and right columns of the partitions respectively correspond to forward and backward propagation.

With the modes defined, the field at the $n$th layer boundary in a finite slab of $L$ layers is expressed as a combination of forward/backward states, originating at layers $n=0, L$ respectively:

$$
\left[\begin{array}{l}
\mathbf{f}^{-}(n) \\
\mathbf{f}^{+}(n)
\end{array}\right]=\left[\begin{array}{l}
\mathbf{F}^{-} \\
\mathbf{F}^{+}
\end{array}\right] \boldsymbol{\Lambda}^{n} \mathbf{c}^{-}+\left[\begin{array}{l}
\mathbf{F}^{+} \\
\mathbf{F}^{-}
\end{array}\right] \boldsymbol{\Lambda}^{L-n} \mathbf{c}^{+} .
$$

In (6), $\boldsymbol{\Lambda}^{n}$ and $\boldsymbol{\Lambda}^{L-n}$ characterise propagation of the mode field through the layer. The modes are normalised to carry unit flux and thus $\mathbf{c}^{\mp}$ represent vectors of modal amplitudes. Analogous expressions to (6) may be written for semi-infinite media $\left(M_{1}\right.$ and $\left.M_{3}\right)$ of Figure 1(a), omitting as necessary either the forward/backward mode train.

Interfacing different media (say $M_{i}$ and $M_{j}$ ) requires the application of continuity conditions at the boundaries and is best represented by interface reflection and transmission matrices which are the matrix analogues of the 
familiar, scalar Fresnel coefficients in optics. To derive their form, we consider a modal field of amplitude $\mathbf{c}_{-}^{i}$ incident from within a semi-infinite region $M_{i}$ giving rise to a reflected field $\mathbf{c}_{i}^{+}$in $M_{i}$ and a transmitted field $\mathbf{c}_{j}^{-}$in $M_{j}$ :

$$
\left(\begin{array}{l}
\mathbf{F}_{i}^{-} \\
\mathbf{F}_{i}^{+}
\end{array}\right) \mathbf{c}_{i}^{-}+\left(\begin{array}{l}
\mathbf{F}_{i}^{+} \\
\mathbf{F}_{i}^{-}
\end{array}\right) \mathbf{c}_{i}^{+}=\left(\begin{array}{l}
\mathbf{F}_{j}^{-} \\
\mathbf{F}_{j}^{+}
\end{array}\right) \mathbf{c}_{j}^{-} .
$$

Solve these equations to deduce the generalised Fresnel matrices $\mathbf{R}_{i j}$ and $\mathbf{T}_{i j}$, respectively defined by $\mathbf{c}_{i}^{+}=\mathbf{R}_{i j} \mathbf{c}_{i}^{-}$and $\mathbf{c}_{j}^{-}=\mathbf{T}_{i j} \mathbf{c}_{i}^{-}$, and which are given by

$$
\begin{aligned}
\mathbf{R}_{i j} & =\left(\mathbf{F}_{i}^{-}\right)^{-1}\left(\mathbf{I}-\mathbf{R}_{j} \mathbf{R}_{i}\right)^{-1}\left(\mathbf{R}_{j}-\mathbf{R}_{i}\right) \mathbf{F}_{i}^{-}, \\
\mathbf{T}_{i j} & =\left(\mathbf{F}_{j}^{-}\right)^{-1}\left(\mathbf{I}-\mathbf{R}_{i} \mathbf{R}_{j}\right)^{-1}\left(\mathbf{I}-\mathbf{R}_{i}^{2}\right) \mathbf{F}_{i}^{-} .
\end{aligned}
$$

In (8) and (9), $\mathbf{R}_{i}=\mathbf{F}_{i}^{+}\left(\mathbf{F}_{i}^{-}\right)^{-1}$ denotes the reflection scattering matrix of a semi-infinite crystal of material $M_{i}$ from free space.

The reflection and transmission matrices $\mathbf{R}$ and $\mathbf{T}$ of the entire structure of Figure 1(a), defined by $\mathbf{r}=\mathbf{R} \boldsymbol{\delta}$ and $\mathbf{t}=\mathbf{T} \boldsymbol{\delta}$, may now be deduced from

$$
\begin{aligned}
\mathbf{r} & =\mathbf{R}_{12} \boldsymbol{\delta}+\mathbf{T}_{21} \Lambda^{L} \mathbf{c}_{2}^{+}, & \mathbf{c}_{2}^{-} & =\mathbf{T}_{12} \boldsymbol{\delta}+\mathbf{R}_{21} \Lambda^{L} \mathbf{c}_{2}^{+}, \\
\mathbf{c}_{2}^{+} & =\mathbf{R}_{23} \boldsymbol{\Lambda}^{L} \mathbf{c}_{2}^{-}, & \mathbf{t} & =\mathbf{T}_{23} \boldsymbol{\Lambda}^{L} \mathbf{c}_{2}^{-},
\end{aligned}
$$

expressing the outgoing wave fields at each interface in terms of reflections and transmissions of incoming wave trains. Solving (10-11) then leads to the reflection and transmission matrices for the composite structure:

$$
\begin{aligned}
\mathbf{R} & =\mathbf{R}_{12}+\mathbf{T}_{21} \boldsymbol{\Lambda}^{L} \mathbf{R}_{23} \boldsymbol{\Lambda}^{L}\left(\mathbf{I}-\mathbf{R}_{21} \boldsymbol{\Lambda}^{L} \mathbf{R}_{23} \boldsymbol{\Lambda}^{L}\right)^{-1} \mathbf{T}_{12}, \\
\mathbf{T} & =\mathbf{T}_{23} \boldsymbol{\Lambda}^{L}\left(\mathbf{I}-\mathbf{R}_{21} \boldsymbol{\Lambda}^{L} \mathbf{R}_{23} \boldsymbol{\Lambda}^{L}\right)^{-1} \mathbf{T}_{12},
\end{aligned}
$$

exhibiting a striking resemblance to the scalar Airy formulation for a three layer Fabry-Perot interferometer. For more complex structures comprising additional layers, the treatment above may be extended by recurrence.

Of interest in the design of $\mathrm{PC}$ devices is their tolerance to fabrication defects, the study of which requires a statistical analysis of ensembles of 
realisations of the exact structure in Figure 1(a). In these, the layers in say $M_{2}$ are not identical and so the analysis requires modification. Instead, we compute matrices $\mathbf{R}_{2}, \mathbf{R}_{2}^{\prime}, \mathbf{T}_{2}, \mathbf{T}_{2}^{\prime}$, respectively denoting the free space reflection and transmission matrices for a composite $M_{2}$ structure from above and below. Matching fields at the $M_{1}-M_{2}$ and $M_{2}-M_{3}$ interfaces, we derive the reflection and transmission matrices $\mathbf{R}$ and $\mathbf{T}$ :

$$
\begin{aligned}
\mathbf{R} & =\left(\mathbf{F}_{1}^{-}\right)^{-1}\left(\mathbf{I}-\mathbf{R}_{23} \mathbf{R}_{1}\right)^{-1}\left(\mathbf{R}_{23}-\mathbf{R}_{1}\right) \mathbf{F}_{1}^{-} \\
\mathbf{T} & =\left(\mathbf{F}_{3}^{-}\right)^{-1}\left(\mathbf{I}-\mathbf{R}_{2} \mathbf{R}_{3}\right)^{-1} \mathbf{T}_{2}\left(\mathbf{I}-\mathbf{R}_{1} \mathbf{R}_{23}\right)^{-1}\left(\mathbf{I}-\mathbf{R}_{1}^{2}\right) \mathbf{F}_{1}^{-} \\
\mathbf{R}_{23} & =\mathbf{R}_{2}+\mathbf{T}_{2}^{\prime} \mathbf{R}_{3}\left(\mathbf{I}-\mathbf{R}_{2}^{\prime} \mathbf{R}_{3}\right)^{-1} \mathbf{T}_{2}
\end{aligned}
$$

\subsection{Photon Conductance}

The concept of photon conductance follows by analogy with the theory of electron conductance [7] in which the conductance is directly proportional to energy transmission. We consider the single guide of Figure 1(b), regarding $M_{1}$ as free space and $M_{2}$ as either a finite slab bounded by free space $M_{3}$, or a semi-infinite medium. The conductance $g$ is defined as the total transmission in all propagating output channels caused by unit input in each of the propagating input channels. Denoting by $\mathbf{T}=\left[t_{p q}^{i j}\right]$, the scattering matrix of the structure with incidence in $M_{i}$ and output in $M_{j}$, with $p$ and $q$ respectively denoting output and input channels, it follows that

$$
g=\sum_{p q}\left|t_{p q}^{i j}\right|^{2}=\operatorname{Tr} \mathbf{T}^{H} \mathbf{T} .
$$

Figure 2 plots the variation of conductance with increasing guide width in a slab of $L=6$ layers, revealing clear "staircase" behaviour which can be understood with a modal analysis similar to that in Section 2.1. Additional conducting modes, which carry energy through the structure, are introduced with increasing guide width, with the 'steps' corresponding to the threshold 

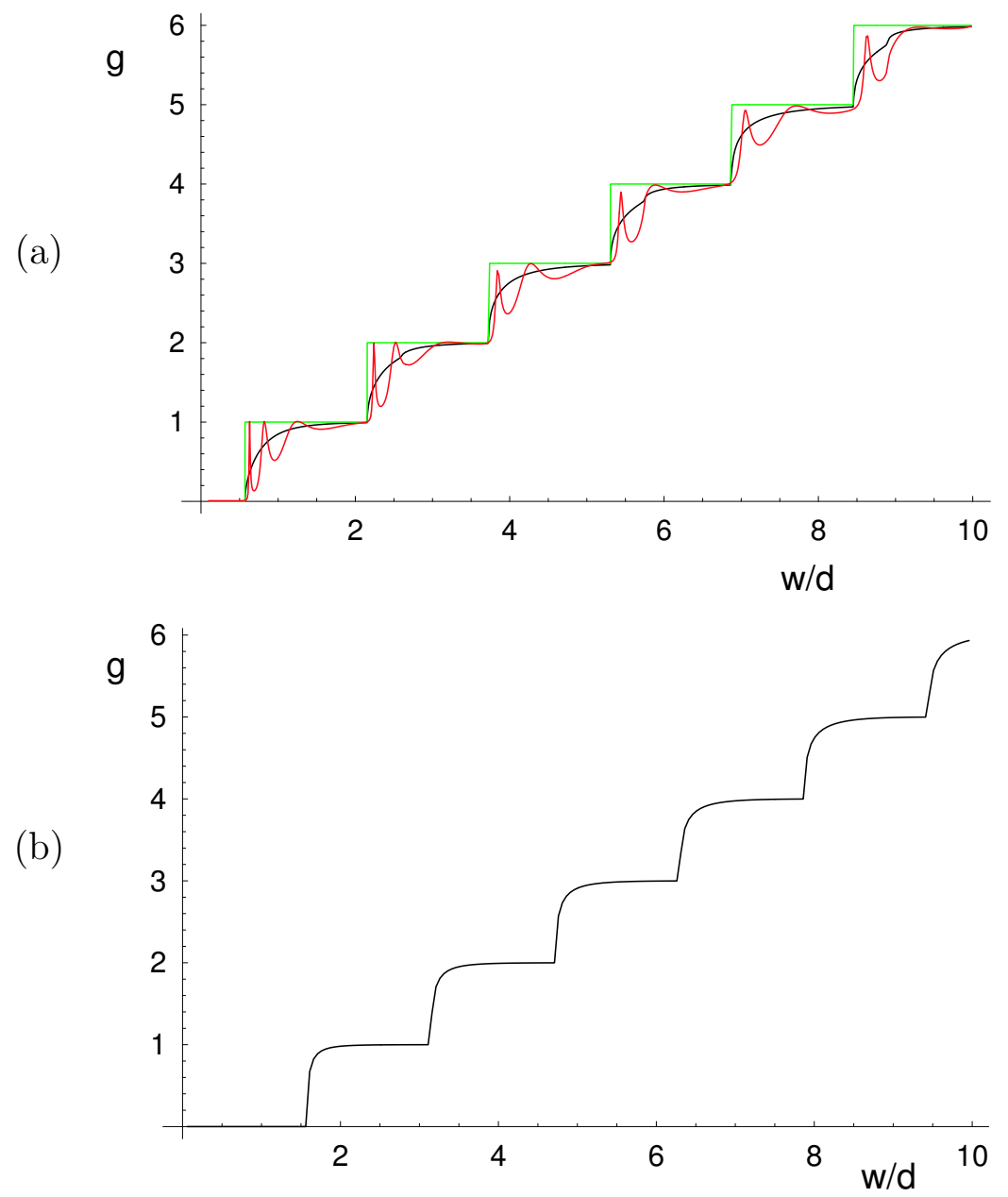

Figure 2: Conductance $g$ versus the guide width $w / d$, normalised to the lattice constant $d$. (a) For a PC guide at $\lambda / d=3.15$ : Green curve, the number of propagating modes; Red curve, conductance for a $L=6$ layer slab; Blue curve: conductance of a semi-infinite PC guide. (b) For a perfectly conducting semi-infinite metal guide for $\lambda / d=3.15$. 
of each new mode. Oscillations in the steps are easily understood in terms of Fabry-Perot resonances between the front and back interfaces of the slab.

The analysis is further enhanced by replacing the slab by a semi-infinite guide (Figure $1(\mathrm{~b})$ ), and exploiting the Reciprocity Theorem to write $g \approx$ $\sum_{m} 1-\left|\rho_{m}\right|^{2}$. The approximate form follows from an asymptotic analysis [2] and characterises accurately the staircase behaviour as the sum over propagating modes of the transmittances $1-\left|\rho_{m}\right|^{2}$ where $\rho_{m} \approx\left[\left(\mathbf{F}_{2}^{-}\right)^{-1} \mathbf{R}_{2} \mathbf{F}_{2}^{-}\right]_{m m}$ and medium $M_{2}$ denotes the semi-infinite guide. The effective reflection coefficient $\rho_{m}$ experienced by mode $m$ at the interface of the guide with free space, diminishes rapidly with increasing guide width, allowing the conductance of each mode to saturate rapidly and carry unit energy (Figure 2(b)).

\section{Computational Results}

In Section 2.2 we computed the conductance of a PC waveguide in the absence of disorder. Realistic devices will have irregularities due to defects of manufacture - for example, perturbations in the positions, radii and refractive indices of cylinders. These may be modelled by the addition to the desired cylinder parameters of random variables drawn from suitable distributions. The effects of such disorder may then be studied using a Monte Carlo approach [8]. We consider an ensemble of PC waveguides in which the $\ell$ th cylinder of a realisation has radius $0.3 d$ and refractive index $n_{\ell}=3.0+\xi$, where $\xi$ is a random variable uniformly distributed in the range $[-Q, Q]$. We then define the level of disorder as $Q / n$, expressed as a percentage, where $n=3.0$ is the cylinder refractive index of the corresponding regular PC waveguide. Figure 3 displays the mean conductance of an ensemble of 100 disordered PC waveguides of length $L=6$. Clearly, the ladder behaviour noted in Figure 2 remains observable with disorder of up to $13.3 \%$, albeit with some degradation, while strong disorder $(26.67 \%)$ destroys the effect. This is consistent with Figure 4 which illustrates the effect of increasing dis- 


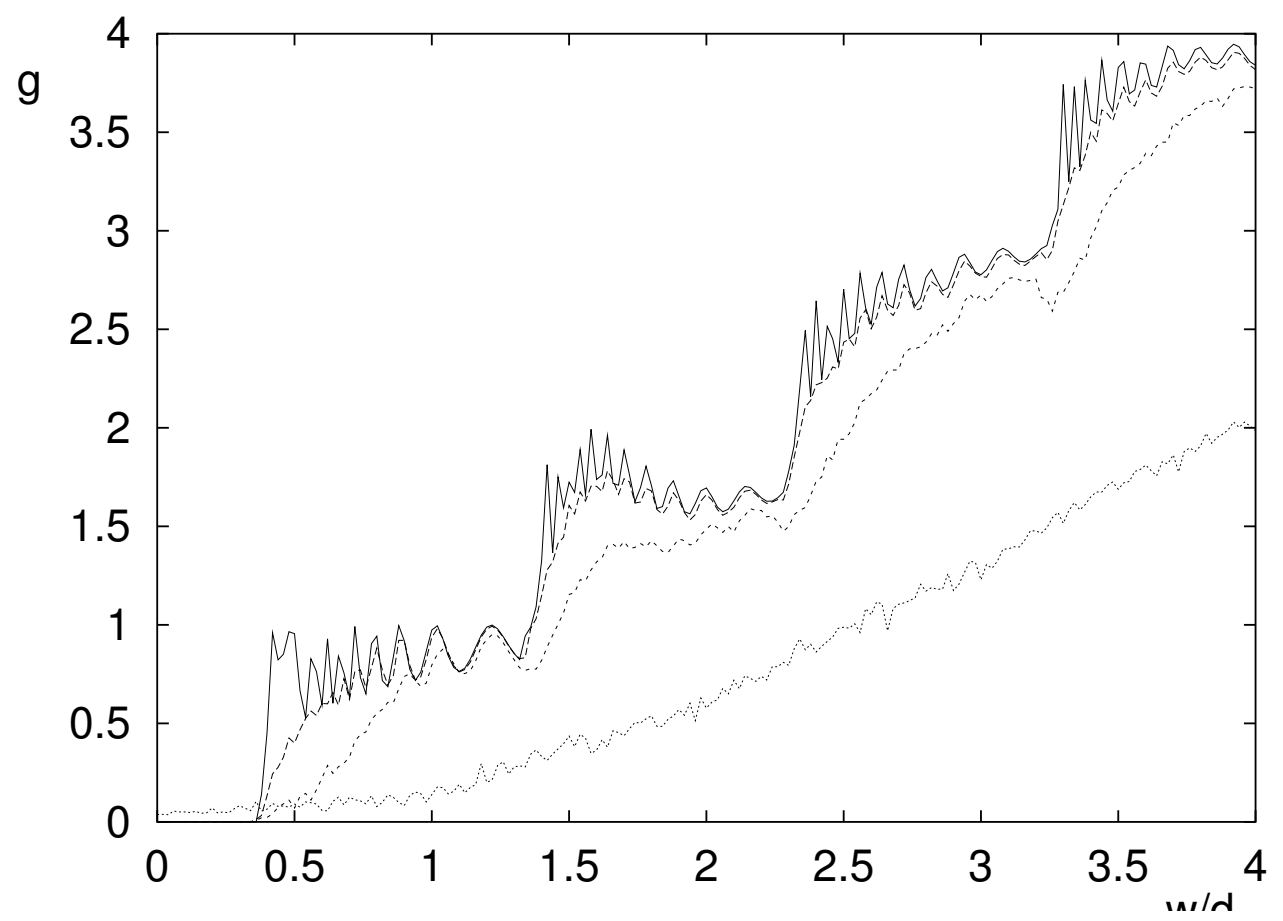

Figure 3: Mean conductance of ensemble of disordered waveguides. As for Figure 2, but with disorder at 0\%, 6.67\%, 13.3\%, 26.67\% from top to bottom. 


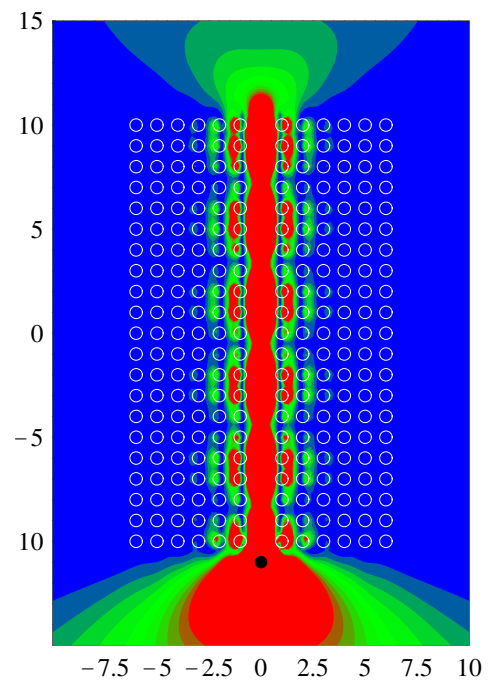

(a)

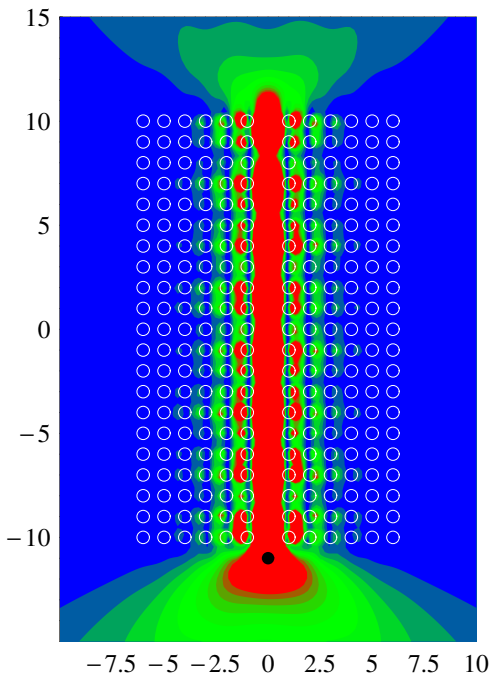

(c)

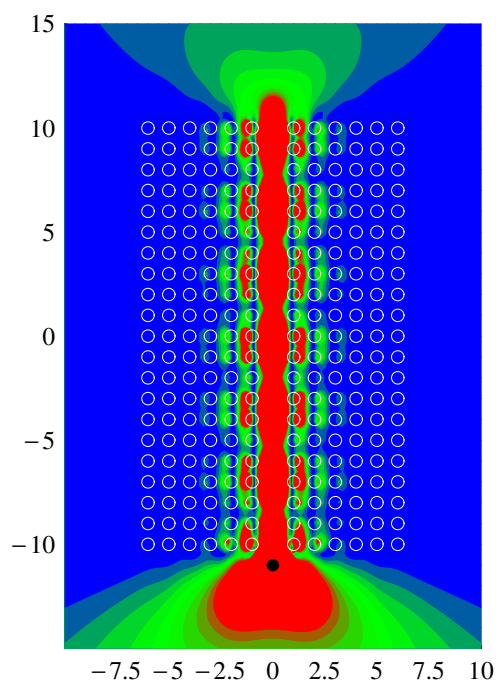

(b)

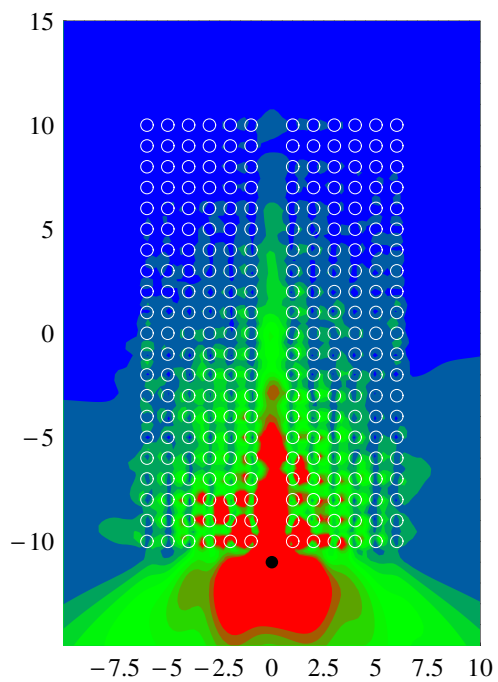

(d)

FIgURE 4: Mean electric field intensity for ensemble of PC waveguides subject to refractive index disorder: (a) $0 \%$, (b) 10\%, (c) 20\%, (d) $30 \%$. 
order in cylinder indices on the mean electric field intensity of ensembles of 48 waveguides of length $L=21$. Each waveguide is embedded in a cluster of 252 cylinders arranged on a square lattice with lattice constant $d$ and is excited by a line source parallel to the cylinder axes radiating at wavelength $3.3 d$. The intensity was computed using the multipole method [1]. The figure indicates substantial robustness of the PC bandgap effect and of localised modes when the cylinders are subjected to random perturbations. In particular we observe little degradation of the localisation effect at $10 \%$ disorder, with some observable degradation at $20 \%$ disorder. At the $30 \%$ level guiding has effectively ceased.

Acknowledgements: The authors acknowledge the support of the Australian Research Council for its funding of the Centre of Excellence for the Ultrahigh-bandwidth Devices for Optical Systems (CUDOs), and also ac3 and APAC for the provision of parallel computing facilities.

\section{References}

[1] A. Asatryan, K. Busch, R. C. McPhedran, L. C. Botten, C. M. de Sterke, and N. A. Nicorovici. Two-dimensional Green's function and local density of states in photonic crystals consisting of a finite number of cylinders of infinite length. Phys. Rev. E, 63(4):046612, 2001. C746, C747, C757

[2] L. C. Botten, A. A. Asatryan, T. N. Langtry, T. P. White, C. M. de Sterke, and R. C. McPhedran. Semi-analytic treatment for propagation in finite photonic crystal waveguides. Opt. Lett., 28:851-853, 2003. C746, C754

[3] L. C. Botten, N. A. Nicorovici, A. A. Asatryan, R. C. McPhedran, C. M. de Sterke, and P. A. Robinson. Formulation for electromagnetic 
scattering and propagation through grating stacks of metallic and dielectric cylinders for photonic crystal calculations. Part I. Method. J. Opt. Soc. Am. A, 17:2165-2176, 2000. C746, C749

[4] L. C. Botten, N. A. Nicorovici, R. C. McPhedran, C. M. de Sterke, and A. A. Asatryan. Photonic band structure calculations using scattering matrices. Phys. Rev. E, 64:046603, 2001. C748

[5] J. D. Joannopoulos, R. D. Maede, and J. N. Winn. Photonic Crystals. Princeton University Press, New Jersey, 1995. C745

[6] S. John. Strong localization of photons in certain disordered dielectric superlattices. Phys. Rev. Lett., 58:2486, 1987. C745

[7] R. Landauer. Spatial variation of currents and fields due to localized scatterers in metallic conduction. IBM J. Res. Develop., 1:223-231, 1957. C752

[8] T. N. Langtry, A. A. Asatryan, L. C. Botten, C. M. de Sterke, R. C. McPhedran and P. A. Robinson. Effects of disorder in two-dimensional photonic crystal waveguides. Phys. Rev. E., 68:026611, 2003. C754

[9] A. Mekis, J. C. Chen, I. Kurland, S. Fan, P. R. Villeneuve, and J. D. Joannopoulos. High transmission through sharp bends in photonic crystal waveguides. Phys. Rev. Lett., 77:3787, 1996. C746

[10] G. H. Smith, L. C. Botten, R. C. McPhedran and N. A. Nicorovici. Cylinder gratings in conical incidence with applications to modes of air-cored photonic crystal fibers. Phys. Rev. E, 66:056604, 2001. C750

[11] E. Yablonovitch. Inhibited spontaneous emission in solid state physics and electronics. Phys. Rev. Lett., 58:2059, 1987. C745

[12] T. P. White, L. C. Botten, C. M. de Sterke and R. C. McPhedran. Ultra-compact resonant filters in photonic crystals. Opt. Lett., 28:2452-2454, 2003. C747 